Precedence-effect thresholds for a population of untrained listeners as a function of stimulus intensity and interclick interval

Kourosh SaberiJoseph V. Antonio

Citation: The Journal of the Acoustical Society of America 114, 420 (2003); doi: 10.1121/1.1578079

View online: http://dx.doi.org/10.1121/1.1578079

View Table of Contents: http://asa.scitation.org/toc/jas/114/1

Published by the Acoustical Society of America 


\title{
Precedence-effect thresholds for a population of untrained listeners as a function of stimulus intensity and interclick interval
}

\author{
Kourosh Saberia) \\ Division of Biology, Caltech, Pasadena, California 91125 \\ Joseph V. Antonio \\ Department of Psychology, California State University, Los Angeles, California 90032
}

(Received 12 September 2001; revised 2 April 2003; accepted 4 April 2003)

\begin{abstract}
Data are reported from 127 untrained individuals under lag- and single-click conditions in a precedence-effect task. In experiment I, each subject completed ten runs in a two-interval forced-choice design under a lag-click condition and three runs under a single-click condition. The cue to be discriminated was an interaural time difference (ITD). Stimuli were 125- $\mu$ s rectangular pulses and the interclick interval (ICI) was $2 \mathrm{~ms}$. Subjects were randomly assigned to three groups of approximately 30. Each group was tested at one stimulus intensity $(43,58$, or $73 \mathrm{~dB})$. Mean threshold within each group was greater than $500 \mu$ s for lag-click ITD conditions, although substantial intersubject variability and a clear effect of stimulus intensity on lag-click ITD thresholds were observed, with lower thresholds for higher intensities. In experiment II, the ICI was varied from 0.3 to $10 \mathrm{~ms}$, and thresholds were obtained from groups of approximately 20 untrained subjects. Data were also collected from three highly experienced observers as a function of ICI. The best naive subject produced mean thresholds near, but not as low as those obtained from experienced subjects. Analysis of adaptive-track patterns revealed abrupt irregularities in threshold tracking, consistent with either losing the cue or listening to the wrong cue in an ambiguous stimulus. (C) 2003 Acoustical Society of America. [DOI: 10.1121/1.1578079]
\end{abstract}

PACS numbers: 43.66.Pn, 43.66.Mk, 43.66.Qp [LRB]

\section{INTRODUCTION}

The precedence effect refers to a variety of onsetdominance phenomena in localization of auditory stimuli (Wallach et al., 1949; Haas, 1949). One defining feature of this effect is a decrease in the ability to perceive spatial information in a sound preceded by another, usually transient, sound. The precedence effect holds for binaural as well as monaural conditions, including along the vertical and frontback axes (Blauert, 1971, 1997; Rakerd et al., 2000). In spite of an extensive history of theoretical (Lindemann, 1986a, b; Zurek, 1980, 1987; Freyman et al., 1997; Saberi, 1996; Hartung and Trahiotis, 2001), neurophysiological (Cranford and Oberholtzer, 1976; Yin and Litovsky, 1995; Mickey and Middlebrooks, 2001), applied (Blauert, 1989; Muncey et al., 1953), and even clinical (Hochster and Kelly, 1981) research, the mechanisms underlying the precedence effect are not well understood. For reviews see Gardner (1968), Zurek (1987), and Litovsky et al. (1999).

Some evidence suggests that the precedence effect is at least partially governed by low-level noncortical processes (Hafter et al., 1983, 1988; Hafter and Dye, 1983; Zurek, 1987; Hartung and Trahiotis, 2001). One such view postulates neural inhibition as a mechanism for reduction of spatial cues (Lindemann, 1986a, b; Hafter et al., 1988), particularly when the lead and lag waveforms contain overlapping spectral energies (Shinn-Cunningham et al., 1995; Saberi,

${ }^{a}$ Current address: Department of Cognitive Sciences, University of California, Irvine, CA 92697. Electronic mail: kourosh@uci.edu
1996). Another approach suggests that peripheral processes including interactions within auditory filters and the interaural phase spectrum can account for many features of the precedence effect, particularly when transient stimuli are employed (Saberi and Perrott, 1995; Tollin and Henning, 1998, 1999; Hartung and Trahiotis, 2001; Zurek and Saberi, 2003). Other evidence, however, points to a complex process involving high-order influences. One striking example is the Clifton effect (1987); Clifton and her colleagues have demonstrated a failure of the precedence effect when the position of the lead and lag sources are reversed between successive presentations (Clifton and Freyman, 1989). These experiments have shown that when the effect fails, it takes several seconds for it to be built up, implicating a slow centralized process. Further evidence against a hard-wired explanation is provided by studies that show learning (Saberi and Perrott, 1990) and top-down influences when cross-correlation cues are ambiguous (Zurek and Saberi, 2003; Saberi and Perrott, 1995; Freyman et al., 1997). Related phenomena in localization, such as the Franssen effect (1960) which demonstrates onset dominance in reverberant environments lasting tens of seconds, are also thought to be driven by such cognitive factors as plausibility (Hartmann and Rakerd, 1989).

Because these diverse findings are inconsistent with a single explanation, current views consider the precedence effect to incorporate several onset phenomena, which in turn has necessitated multiple approaches to its study (Blauert, 1997; Djelani and Blauert, 2001); The reader is referred to Blauert and Col (1991) for a discussion of irregularities in 
defining the precedence effect. The current study uses a population approach to study precedence in which we examine thresholds from a large number of untrained listeners. The study was motivated by observations of large variability across threshold estimates in several experiments, particularly from naive subjects, that appear statistically nonstationary and difficult to attribute to sensory inhibition (Saberi and Perrott, 1990; Zurek and Saberi, 2003; Tollin and Henning, 1998, 1999), and by discrepant findings between Saberi and Perrott (1990) and Litovsky et al. (2000) related to the effects of practice on lag-click ITD discrimination.

In the current study, we examined population thresholds for two-transient dichotic stimuli. The population was composed of experimentally naive young college students. This approach allowed the establishment of a databank as well as a baseline to compare with thresholds from experienced subjects. In the current experiments, thresholds were obtained from 127 individuals, grouped randomly into sets of approximately 20 to 30 . We selected this group size because it provided sufficient statistical power for estimation of group parameters (Pitman, 1993; Hays, 1981). For each group, we obtained interaural delay thresholds as a function of interclick interval (ICI) and stimulus intensity because intensity has previously been shown to affect lag-click thresholds (Goverts et al., 2000). Finally, we explored irregularities in patterns of responses observed in adaptive tracks. Analysis of tracks revealed unstable psychometric functions, predominately for naïve listeners, but also for experienced subjects, implicating a possible influence of conflicting stimulus cues.

\section{EXPERIMENT I: INTERAURAL DELAY THRESHOLDS FOR SINGLE AND LAG CLICKS AS A FUNCTION OF STIMULUS INTENSITY}

\section{A. Method}

Stimuli were $125-\mu$ s rectangular pulses generated by a Dell PC (OptiPlex GX1) and presented through 16-bit digital-to-analog converters (Sound Blaster Live, Milpitas, CA) at a sampling rate of $40 \mathrm{kHz}$ and were low-pass filtered at $20 \mathrm{kHz}$. Subjects listened to stimuli over Sony (MDR-V1) headphones in an acoustically isolated steel chamber (Industrial Acoustics Company; interior dimensions of $1.8 \times 1.9$ $\times 2 \mathrm{~m}^{3}$ ). The level of a single pulse was calibrated to 43,58 , or $73 \mathrm{~dB}$ (A weighted, slow time average), depending on the experimental condition, using a 6-cc coupler, 0.5-in. microphone (Brüel \& Kjær, Model 4189), and a modular precision sound analyzer (Brüel\&Kjær, Type 2260). ${ }^{1}$ Measurements of the headphone outputs, using a 6-cc coupler, 0.5-in. microphone $(\mathrm{B} \& \mathrm{~K})$, a conditioning amplifier (Nexus, B\&K), and an analog-to-digital (A/D) converter (Sound Blaster), showed that the pulse spectrum was linear for the three intensities tested $(43,58$, and $73 \mathrm{~dB})$. The timing between pulses and between channels, as well as the level between channels, were checked for accuracy at each SPL with a dual-channel digital storage oscilloscope (Tektronix, Model TDS210) and the microphone assembly described above. Measurements showed that for all three stimulus intensities, the interclick interval for a two-click train with an ICI of 2 ms, measured at the output of the headphones, was unaf- fected by the chosen intensities and the headphone transfer function. Timing between channels was also checked at the output of the headphones by placing a microphone equidistant from the two headphone channels and recording dichotic pulses with different delays between channels. This measurement showed that between-channel timing was precise at the three tested intensities.

Subjects were untrained young college students (ages 18-22) who were recruited from campus advertisements and were paid an hourly wage for their participation. In addition to an hourly wage, subjects were told that the individual with the lowest overall threshold within their respective group (of approximately 30 subjects) would receive a financial bonus. All subjects had normal hearing based on self-report. All subjects were asked if they had a head cold or congestion, and, if so, were rescheduled. Subjects listened to each task in an initial pilot run in the presence of the experimenter for several trials until the experimenter was satisfied that the subject understood the task. This short pilot run was then terminated, usually within 15 trials, and the experiment was started after the experimenter left the chamber. Each subject completed a total of 13 runs. Each run consisted of 50 trials in an adaptive two-down one-up design which tracks the subject's 70.7\% correct-response threshold (Wetherill and Levitt, 1965; Levitt, 1971). The first two runs, as well as the last run, measured the subjects' threshold for a single dichotic click; this was a control condition to which thresholds from the lag-click conditions were to be compared. In runs $3-12$, the stimulus in each interval of a trial consisted of two clicks, the first representing the lead diotic event and the second representing the lag dichotic event. The ICI was 2 ms. This value has been shown to produce a strong precedence effect for impulsive sounds (Wallach et al., 1949; Zurek, 1980; Zurek and Saberi, 2003), and was selected to be near the training value used by Saberi and Perrott (1990).

On the first interval of each trial of the single-click condition, the dichotic click led to one randomly selected ear by an ITD and, in the second interval, it led to the other ear by the same ITD. The subject's task was to determine if the order of leading ITD was left-ear then right-ear or vice versa. Perceptually, this would be equivalent to determining if the two intracranial sound images in the two intervals of the trial were heard left then right, or right then left. The subject would then press either a left or a right key to respond (leftkey response meant that they perceived the sound orders as right to left). Visual feedback was provided after each trial in two forms. First, the subject was informed if s/he was correct. Second, in an image window on the screen, the adaptive track for the current run was displayed which included the ITD values up to the current trial in a graph (i.e., a plot of the trial number versus ITD value). Subjects were instructed to use this trial-by-trial updated graph and the feedback to try to achieve the lowest possible score. A horizontal baseline at 75 $\mu$ s was plotted on this graph as a target level, and subjects were instructed to attempt to reach values below this line. The initial value of the total ITD on each run was $1300 \mu \mathrm{s}$, i.e., $650 \mu \mathrm{s}$ in each interval. Two successive correct responses led to a reduction of the total ITD by a stepsize of $0.2 \log$ units until the fourth reversal and $0.05 \log$ units 
thereafter, rounded to the nearest $25 \mu \mathrm{s}$ [e.g., an ITD of 1300 $\mu \mathrm{s}$, after two successive correct responses, would be reduced to $10^{\log (1300)-0.2}=820 \mu \mathrm{s}$; see Saberi (1995)]. An incorrect response led to an increase in total ITD by the stepsize. The $25-\mu$ s resolution was determined by the sampling rate of 40 $\mathrm{kHz}$. The variance added from this minimum stepsize to threshold measurements was negligible. Simulations showed that using a $25-\mu$ s stepsize increases the standard deviation of threshold estimates by a maximum of $12.5 \mu \mathrm{s}$. Lowest and highest possible values of ITD within an interval were 0 and $650 \mu \mathrm{s}$, and if the adaptive track required values outside this range, they were corrected to these floor and ceiling values. In all cases, threshold was estimated as the average of the stimulus values at track reversal points, after the fourth reversal. Usually, five to eight reversals went into the calculation of each threshold value. In runs 3-12, the stimulus within each interval consisted of two clicks, a lead diotic click and a lag dichotic click with an ICI of $2 \mathrm{~ms}$. The ITD of the lead click was always zero, and thus carried no information for performing the task. The ITD of the lag click was varied according to the adaptive rules described above.

\section{B. Results}

Figure 1 shows results from experiment I. Data from 89 subjects are shown, with 30,29 , and 30 subjects for each row of panels from top to bottom, respectively. Each row shows data for one stimulus intensity. Left panels show threshold interaural delays for a single click, and the right panels are thresholds for the lag click in a two-click design. Each histogram in the right panels represents approximately 300 threshold estimates, i.e., 10 per subject, and each histogram on the left is based on approximately 90 threshold estimates, i.e., 3 per subject. The bin width is $100 \mu$ s. Arrows show mean threshold within each panel.

The average population thresholds for the three stimulus intensities of 73, 58, and $43 \mathrm{~dB}$ are 247, 275, and $286 \mu \mathrm{s}$, respectively, for the single-click condition, and 535, 822, and $838 \mu$ s for the lag-click condition. These threshold values from untrained subjects are quite high, but also vary a great deal both across and within subjects as will be discussed in a later section. The histogram modes for the single-click condition are somewhat smaller, being $150 \mu$ s for all three intensities. For the lag condition, however, the modes are 1050 $\mu$ s for the two lower intensities, and a considerably smaller value of $150 \mu \mathrm{s}$ for the 73-dB intensity. The upper mode of $1050 \mu$ s represents the limits imposed on the adaptive track. While the maximum ITD was $1300 \mu \mathrm{s}$, the effective ceiling was $1050 \mu \mathrm{s}$. Simulations showed that the occurrence of two successive correct responses by chance on a proportion of trials will lead to an effective ceiling of approximately 1050 $\mu \mathrm{s}$, even if the cue is undetectable.

There is a striking difference between the effects of intensity on single- and double-click conditions. The maximal percentage increase in mean threshold across intensities is $16 \%$ for the single-click condition, and 57\% for the lag-click condition. Even greater is the difference between the modes of the distribution of thresholds. For the single-click condition, there is a no shift in the mode, while for the lag-click condition there is a $700 \%$ decrease from the lowest to the
Single Click
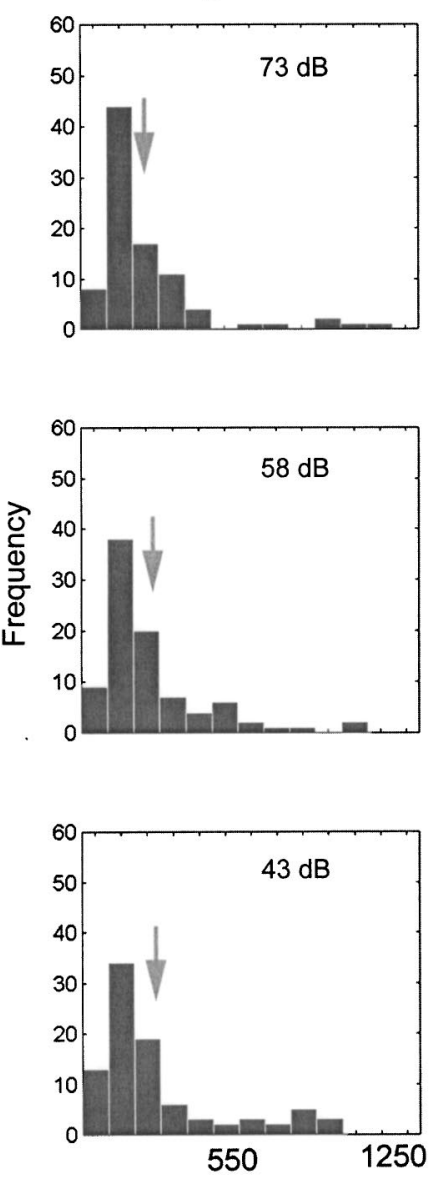

ITD Threshold ( $\mu$ sec)
Lag Click
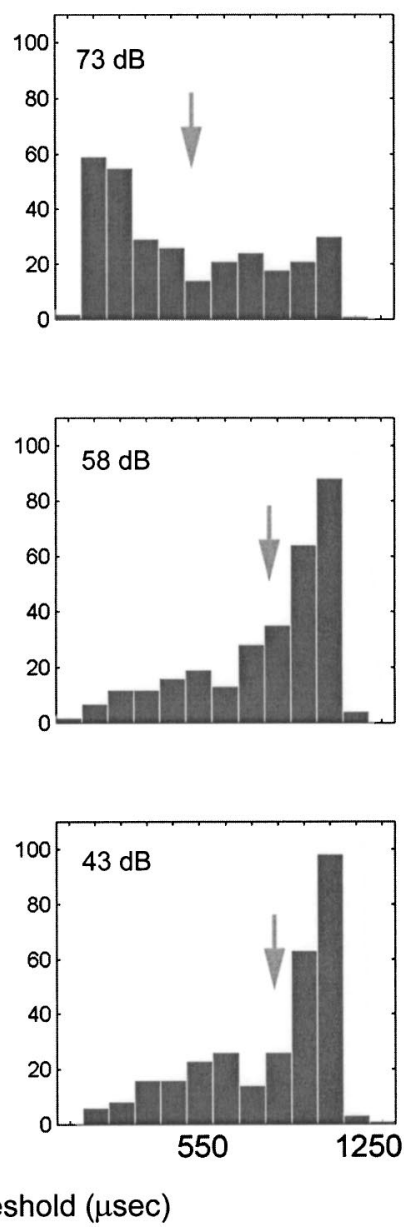

FIG. 1. Results from experiment I. Histograms of interaural delay thresholds in single- and lag-click conditions as a function of stimulus intensity. Arrows show mean thresholds. Data are from 89 subjects.

highest intensities and virtually all of this shift occurs between the two highest intensities. Consistent with these observations, Goverts et al. (2000) have shown that the precedence effect is most effective for mid-range stimulus intensities.

\section{EXPERIMENT II: EFFECTS OF INTERCLICK INTERVAL}

\section{A. Method}

All methods in this experiment were the same as in experiment I, except that the ICI was a parameter of study and a single threshold at each value of ICI was obtained from each subject. The ICIs were $0.3,1.0,1.25,1.75,2,2.35,5$, and $10 \mathrm{~ms}$. Within a run, the value of ICI was fixed. Only two stimulus intensities, 73 and $58 \mathrm{~dB}$, were examined. For the 73-dB condition, thresholds were obtained from 20 subjects, and for the 58-dB condition, from 18 subjects. The subjects differed from those used in experiment I. Data were also obtained from these subjects in a single-click control condition, for which two threshold estimates were obtained and averaged. The single-click conditions were run at the beginning and end of the session. The order in which each subject ran each of the ICI conditions was randomized. 

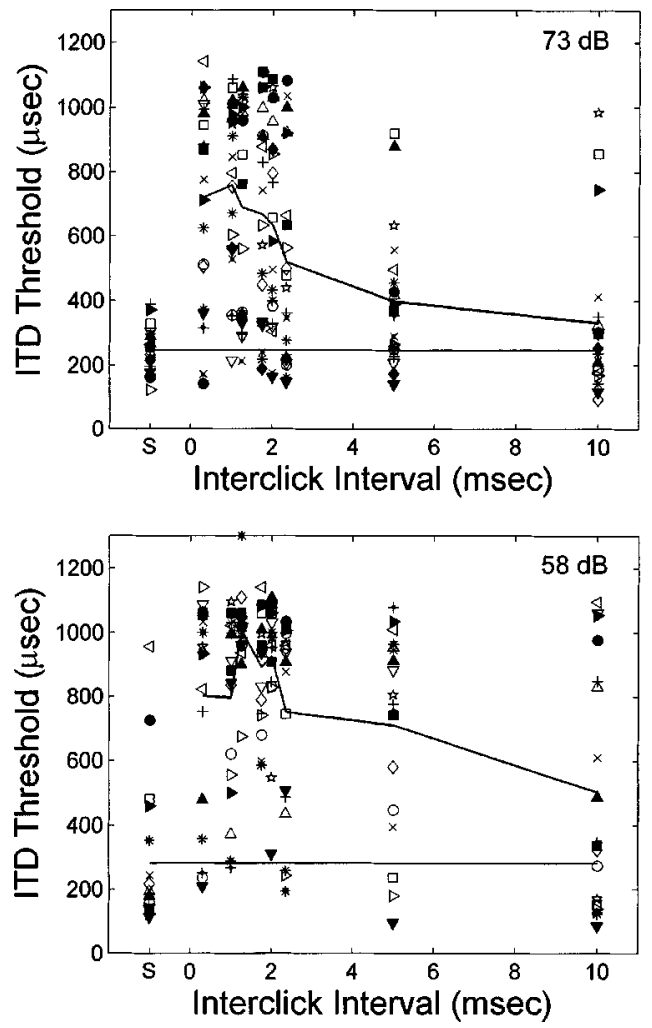

FIG. 2. Results from experiment II. ITD thresholds as a function of interclick interval. Each symbol represents data from one subject, and the solid curve is the mean threshold. The single-click thresholds are plotted on the left of each graph, and the mean single-click threshold is indicated by the horizontal line extending across the graph to facilitate visual comparison. Top and bottom panels shows data from 20 and 18 subjects, respectively, measured at two stimulus intensities.

\section{B. Results}

Figure 2 shows results from experiment II. The top panel shows ITD thresholds from individual subjects (different symbols) as a function of ICI at a stimulus intensity of 73 $\mathrm{dB}$, and the lower panel shows thresholds for an intensity of $58 \mathrm{~dB}$. The symbols on the left in each panel show data for the single-click control, and the mean single-click threshold is indicated by the horizontal line extending across the graph to facilitate visual comparison. No significant difference was observed between the two single-click runs at the beginning and end of the run [Wilcoxon nonparametric matched-pairs test $\mathrm{W}(18)=56$, ns for the $58-\mathrm{dB}$ intensity and $\mathrm{W}(20)=62$, $\mathrm{ns}$ for the $73-\mathrm{dB}$ intensity]. The upper curve in each panel shows the mean threshold across all subjects. Note the wide range of thresholds across subjects. The lower panel shows that thresholds for the 58- $\mathrm{dB}$ condition are generally similar in form, but higher in value, relative to the 73-dB condition. Thresholds, as expected, are a nonmonotonic function of ICI with a lower mean threshold for the ICI of $0.3 \mathrm{~ms}$ compared to the peak of the function at an ICI of 1 . The reduced strength of precedence at very low ICIs is typically referred to as "summing localization" (Leakey, 1957; Blauert, 1997).

\section{EXPERIMENT III: COMPARISON TO THREHSOLD FROM EXPERIENCED SUBJECTS}

In this section we compare thresholds from the population of untrained subjects to those from highly experienced subjects. Previous work has shown that rigorous training leads to improvements in detection of an ITD in the lag click of a two-transient stimulus (Saberi and Perrott, 1990). Others, however, have reported that training has no effect on ITD thresholds in a precedence-effect task (Litovsky et al., 2000). Here we report thresholds for three experienced subjects and show long-term training effects for one subject. The purpose was not an extensive study of training, but to gain better insight into why some subjects may produce significantly lower thresholds than others.

\section{A. Method}

Three experienced subjects were used, two having over $10 \mathrm{~h}\left(\mathrm{~S}_{\mathrm{e}} 1, \mathrm{~S}_{\mathrm{e}} 2\right)$ and the other $\left(\mathrm{S}_{\mathrm{e}} 3\right) 50 \mathrm{~h}$ of training on precedence-effect tasks. All three had over $100 \mathrm{~h}$ of training on other lateralization and localization tasks prior to the start of this experiment. Data from a fourth subject $\left(\mathrm{S}_{\mathrm{e}} 4\right)$ with 66 $\mathrm{h}$ of training on the precedence effect will also be described separately. Subject $\mathrm{S}_{\mathrm{e}} 1$ was a 20 -year-old male, $\mathrm{S}_{\mathrm{e}} 2$ was a 25-year-old female, and $\mathrm{S}_{\mathrm{e}} 3$ was a 37-year-old male. $\mathrm{S}_{\mathrm{e}} 3$ was one of the subjects used in Saberi and Perrott (1990) and is the first author. Subject $\mathrm{S}_{\mathrm{e}} 4$ was a completely naive 21-yearold female listener who was selected for training because her thresholds from experiment I were among the highest of all subjects tested. The stimulus level was $73 \mathrm{~dB}$. The task and stimuli were the same as those described for experiment II. As further controls, the ICI was jittered from interval-tointerval by a random value of up to $10 \%$ to distort possible monaural pitch cues, and, in addition, no feedback was provided as to the correctness of the responses. The paradigm used in the first two experiments is referred to as a commutative design. Here, in addition to the commutative design, a second paradigm referred to as a center-side paradigm is used. This paradigm is the same as that employed by Saberi and Perrott (1990) in which both clicks in the first interval of a trial were diotic. In the second interval, the first click was diotic and the second click had an ITD to be detected, either leading to the left or right ear.

\section{B. Results}

The upper curves in both panels of Fig. 3 show the curve replotted from the upper panel of Fig. 2 (untrained subjects, commutative task). Error bars represent \pm 1 standard error of the mean. The lower curves in the top panel show thresholds from two experienced subjects $\left(\mathrm{S}_{\mathrm{e}} 1\right.$ and $\left.\mathrm{S}_{\mathrm{e}} 3\right)$ in the commutative design. These curves represent single-run threshold ITDs in the lag-click condition as a function of ICI. The symbols to the left in each panel show ITD thresholds for the single-click control. Thresholds from experienced subjects, measured using the commutative paradigm, have a peak of about $150 \mu$ s, slightly higher than values reported by Saberi and Perrott (1990) who employed a center-side paradigm. We replicated the center-side paradigm, and these results, from three experienced subjects $\left(\mathrm{S}_{\mathrm{e}} 1, \mathrm{~S}_{\mathrm{e}} 2\right.$, and $\left.\mathrm{S}_{\mathrm{e}} 3\right)$, are shown in the bottom panel of Fig. 3.

Thresholds obtained from experienced subjects in the center-side paradigm were consistently below $100 \mu$ s at all ICIs, are more similar in magnitude to the reported thresholds in Saberi and Perrott (1990), and are significantly 

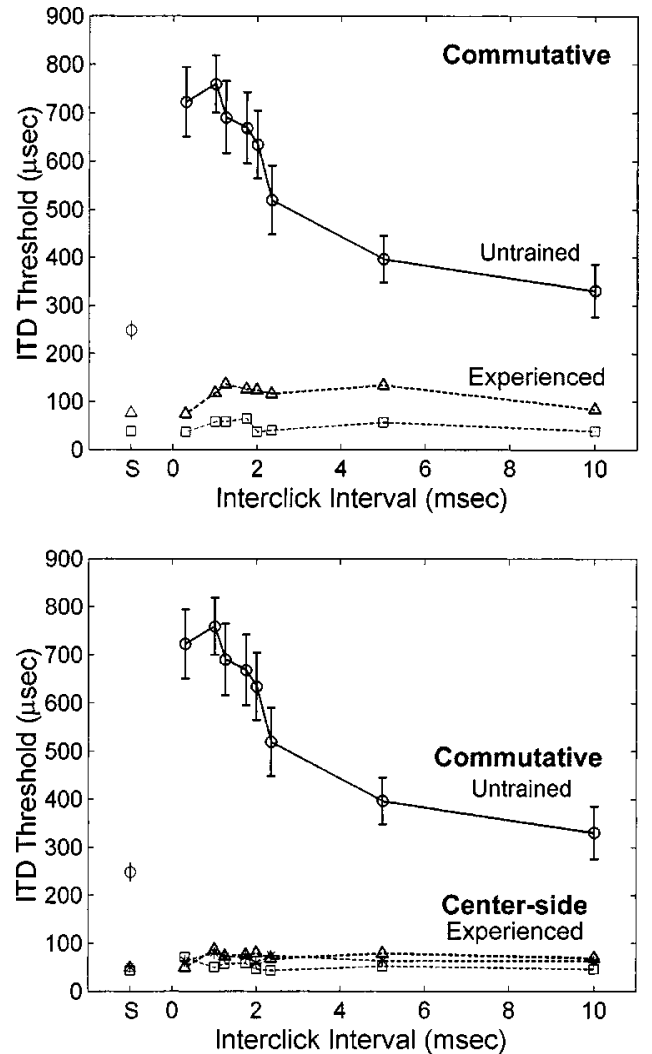

FIG. 3. Lower curves in each panel show thresholds from experienced observers, and the upper curve is the population mean replotted from Fig. 2. Error bars represent \pm 1 standard error of the mean. The symbols to the left of each panel show thresholds for single-click conditions. Top and bottom panels show data collected from experienced subjects using the commutative and center-side designs, respectively (see text). Data from untrained subjects in both panels were collected using the commutative design. smaller than those reported by Litovsky et al. (2000) who also used the center-side design. It is interesting that the center-side design has produced smaller thresholds compared to the commutative design. If one assumes that the presence of an interaural disparity causes an increase in the variance of the interaural cues within the internal composite stimulus, and not always a consistent lateralization, then it may be easier to compare a stimulus that contains an interaural disparity to a diotic stimulus than to compare two stimuli with interaural delays. ${ }^{2}$

A detailed examination of the adaptive tracks for experienced and untrained subjects revealed unexpected and informative patterns. Panel (a) of Fig. 4 shows thresholds from one experienced subject $\left(\mathrm{S}_{\mathrm{e}} 1\right)$ in the center-side paradigm. During this single run of threshold estimates as a function of ICI, this subject showed very low thresholds at all ICIs, except at an ICI of $1.25 \mathrm{~ms}$, for which the threshold was over $300 \mu$ s (upper asterisk). When we inquired, this subject reported that he perceived "reversals" during that particular run, in that the side to which the stimulus ITD led was opposite to what he perceived on a subset of trials (the interaural delay became larger even though the subject was convinced that he was responding correctly). The subject repeated this single condition on the very next run, and showed threshold improvements of $459 \%$ (lower asterisk), from a value of 340 to $74 \mu \mathrm{s}$. This improvement occurred within one run. ${ }^{3}$ We inquired if the subject had been inattentive during the high-threshold run, and the subject was insistent that he was fully attentive and perceived a reversed cue.

Panel (b) of Fig. 4 shows the adaptive track for the highthreshold run by this subject [upper asterisk in (a)], and panel (c) shows the adaptive track for the low-threshold run [lower asterisk in (a)]. These tracks are instructive in that they show
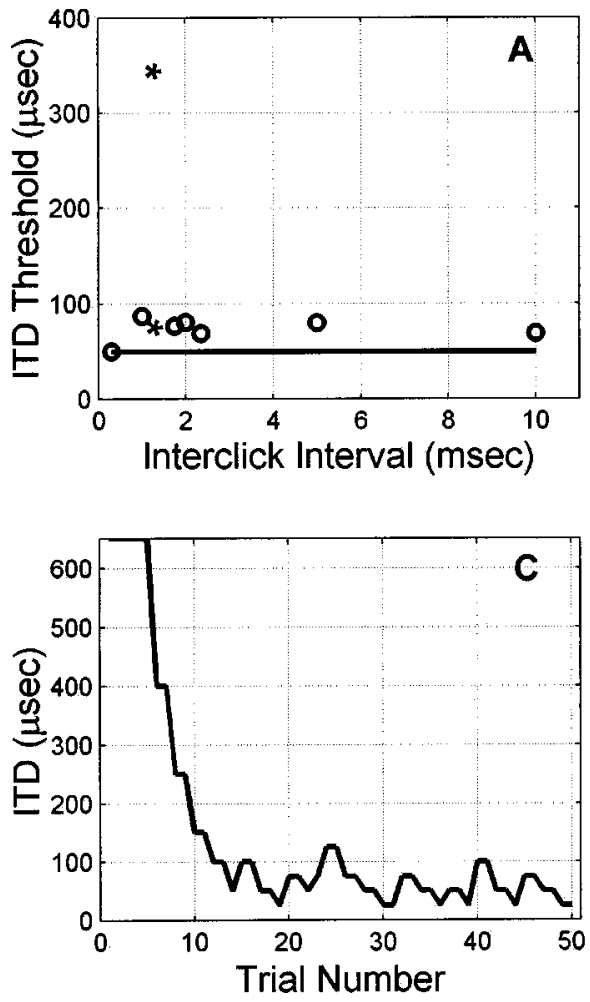
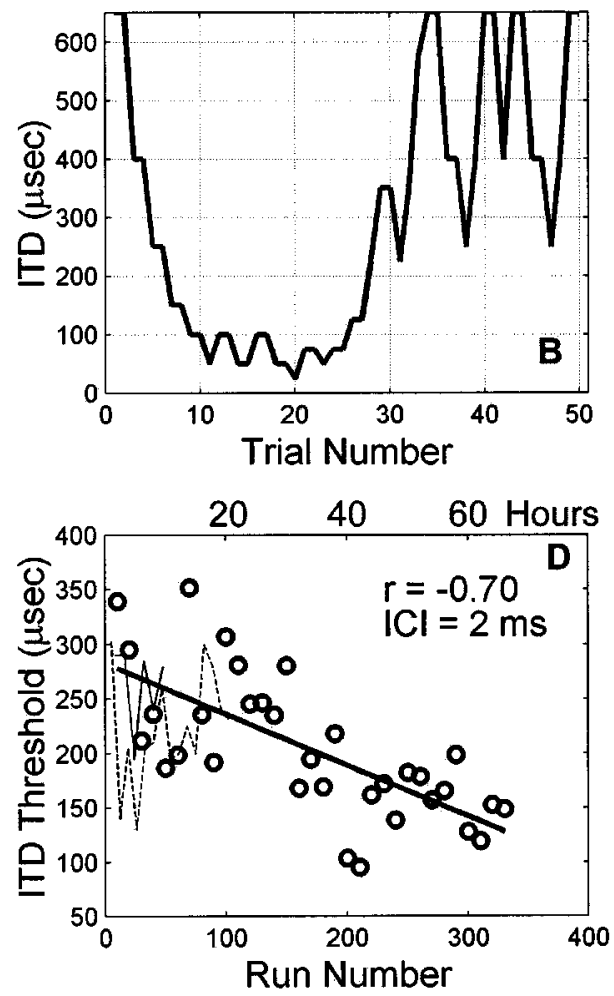

FIG. 4. (a) data from one experienced subject $\left(\mathrm{S}_{\mathrm{e}} 1\right)$. Each circle represents one threshold run in the center-side design. The asterisks are thresholds from two consecutive runs at the same ICI of $1.25 \mathrm{~ms}$. A $459 \%$ improvement in threshold is observed within one run. (b) Adaptive track from the upper asterisk in (a). (c) Adaptive track from lower asterisk in (a). (d) Training data from subject $\mathrm{S}_{\mathrm{e}} 4$ grouped into ten runs per point. The lower abscissa shows the number of runs and the upper abscissa shows the number of hours of training. The fitted line is a linear regression. The dashed curve and solid curve are data of subjects S3 and S7 from Litovsky et al. (2000). 
that the high threshold is a result of averaging very low values of the stimulus with very high values. This pattern is unlike what is expected from a subject with a high sensory threshold and implies a dual process: at the early stages, the ITD cue is detectable whereas at the late stages of the track, consistent with the subject's report, an apparent reversal of the percept or possibly a loss of the signal cue is suggested. On the next run at this ICI, the track shows a consistently low trajectory [panel (c)]. As will be discussed later, tracks such as that shown in panel (b), which were numerous for the untrained subjects, suggest a nonstationary discrimination process.

In general, the intersubject variability was quite high. Some subjects showed moderately low thresholds from the start and others showed quite high thresholds. The experienced subjects had prior extensive experience in lateralization tasks and had shown low thresholds from the beginning of the current experiment. It is therefore difficult, at least for two of the experienced subjects, to determine whether their low lag-click thresholds are a result of extensive prior exposure to lateralization and precedence tasks or having a priori low-threshold.

For comparison, we selected one of the poorest performing subjects from the pool of all untrained subjects. We then trained this subject at an ICI of $2 \mathrm{~ms}$ for several months. In addition to an hourly wage, the subject was given a financial reward whenever the averaged threshold for a 2-h session was lower than all previous sessions. The training data for this subject is shown in panel (d) of Fig. 4, grouped into runs of 10 . After 330 runs $(\sim 66 \mathrm{~h})$ in the commutative design, it is apparent that the subject's thresholds had improved to among the best of the group. The subject could not continue the training because of reasons unrelated to the experiment. It is evident that even after $66 \mathrm{~h}$ of training, the average thresholds for this subject had not yet reached a lower asymptote. If the performance of other experienced subjects is an indication, this subject's final threshold estimates would have likely been even lower if measured in a center-side design. For comparison, we have also plotted training data from subjects S3 and S7 of Litovsky et al. (2000), who produced high thresholds from the start, were inexperienced in lateralization tasks, and maintained a high threshold level for 10 to $20 \mathrm{~h}$ [thin dashed and solid lines in Fig. 4(d)]. It would be difficult to see an improvement for subject $\mathrm{S}_{\mathrm{e}} 4$ from the current study if the data are limited to initial $20 \mathrm{~h}$ of training.

\section{EVIDENCE FOR UNSTABLE TRACK TRAJECTORIES}

The track shown in panel (b) of Fig. 4 prompted us to examine the adaptive tracks from the population of untrained subjects. Such an abrupt change in track trajectory, seen only for lag-click conditions, is indicative of an unstable psychometric function that is, possibly, a composite of a dual process. We speculate that one process is based on a low sensory threshold, and a second process is based on either a cue reversal or loss of the primary signal cue.

An inspection of tracks from untrained subjects showed that on a significant proportion of runs $(\sim 20 \%)$ that used short ICIs $(<5 \mathrm{~ms})$ irregular patterns uncharacteristic of a single stable cue were observed. ${ }^{4}$ Such irregular patterns were rarer or nonexistent for the three experienced subjects, with the only instance being that shown in Fig. 4(b). Six irregular tracks from six untrained subjects, grouped into two types of patterns, are shown in Fig. 5. Other interesting patterns also existed that are not shown, such as two-peak or single-trough patterns. The left panels of Fig. 5 show U-shaped trajectories which, until near the end of the run, are typical of a low-threshold run, and are elevated at the end. The right panels show performance consistent with a low threshold at the beginning and end of the track, and an abrupt elevation of threshold at the middle part. The horizontal line within each panel is the $70.7 \%$ threshold for that run.

\section{DISCUSSION}

The current study investigated lateralization performance in a precedence effect task for a population of subjects with no prior experience in studies of sound localization. Because previous studies have reported large disparities in thresholds (Saberi and Perrott, 1990; Litovsky et al., 2000; Gaskell, 1983; Zurek, 1987; Perrott et al., 1989; ShinnCunningham et al., 1993; Yost, 1984) baseline data were collected from large groups of untrained individuals. Population ITD thresholds for single- and lag-click conditions showed high intersubject variability and dependence on intensity.

A comparison of within- to across-subject variability revealed differences between categories of subjects, in that some subjects from the outset displayed considerably lower thresholds than others. A small number of naive listeners consistently produced low lag-click thresholds. Others produced generally low thresholds interspersed with one or two high values, and still another group consistently produced high thresholds $(>400 \mu \mathrm{s})$ similar to some early studies (Zurek, 1980; Gaskell, 1983). Individuals who were experienced in lateralization showed low lag-click thresholds less than $100 \mu \mathrm{s}$ at all ICI in the center/side task and less than $150 \mu \mathrm{s}$ in the commutative design. In addition, one subject who was not experienced in lateralization and who showed variable thresholds that on average were among the highest of all subjects, during months of training, showed a gradual decline in ITD threshold in the commutative task, suggestive of a slow learning process for this subject.

Several factors may have contributed to the observed low thresholds for some subjects. First, a subject's previous exposure to lateralization tasks in general, including precedence-effect tasks, may be important. Second, some subjects from the start display low pretraining thresholds, whether experienced or not. Third, data from one subject suggests that some subjects with high initial thresholds may improve with extensive training. Fourth, low lag-click thresholds may also be related to stimulus features such as intensity, or to experimental design factors such as financial incentive for improved performance.

Wright and Fitzgerald (2001) have recently reported data on learning in binaural tasks that may have bearing on the present study. They have distinguished between two learning processes: a rapid learning effect that occurs within 

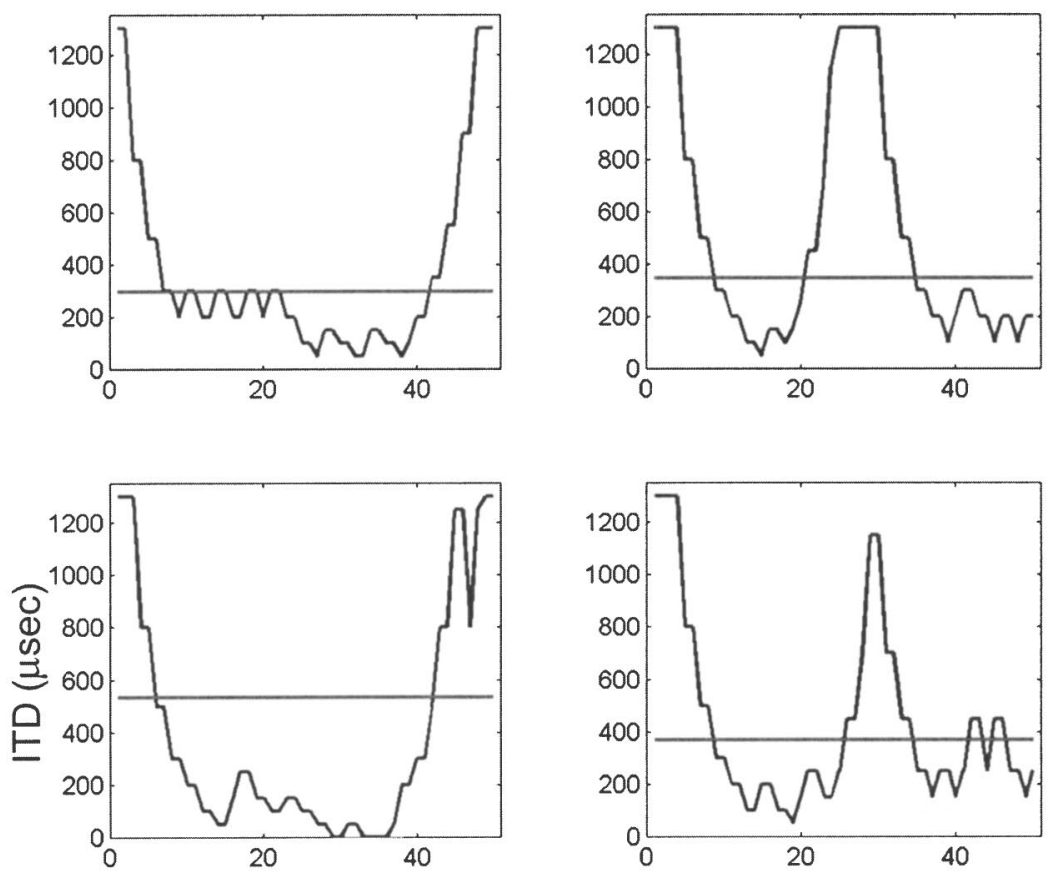

FIG. 5. Sample adaptive tracks from six untrained subjects indicative of a nonstationary lag-click threshold (see text). Solid line shows estimated ITD threshold from the track.
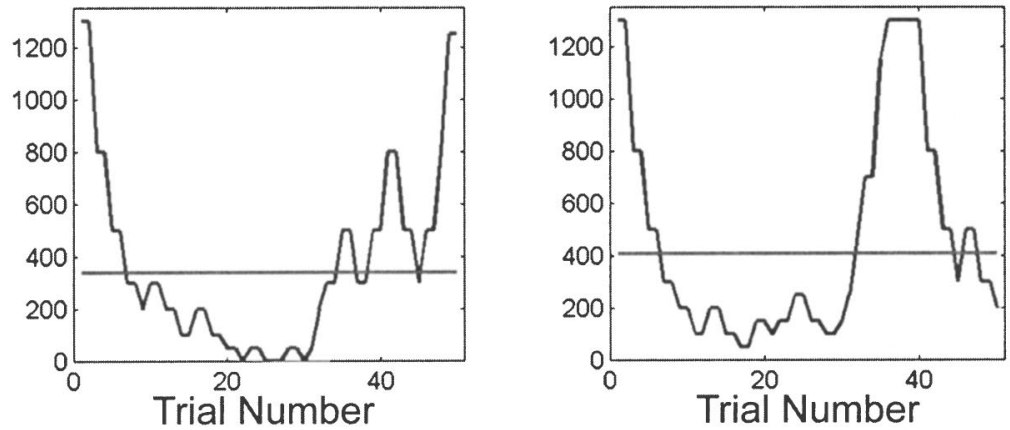

the first few sessions and attributed to procedural learning, and a more gradual learning process attributed to fundamental changes in stimulus processing. The former effect was observed for both ITD and ILD cues, but the latter only for ILD processing. This finding appears different than ours [Fig. 4(d)] and Hafter and Carrier (1970) who show longterm learning for tasks employing ITDs, but similar to others who have reported no long-term learning with low-frequency masking-level-difference or simple ITD discrimination tasks (Bernstein et al., 1998). A close inspection of the individual subject data of Wright and Fitzgerald (2001) shows that although mean thresholds across subjects does not support long-term changes in ITD thresholds, some subjects did display either a long-term gradual decrease in ITD thresholds (their Fig. 1, subjects L9, L10) or a markedly lower posttraining threshold (L12, L14). This suggests that even in a simple ITD-discrimination task, intersubject variability in learning may exist (also see subjects L7 and L8 who do not display long-term learning in an ILD-discrimination task). Given the intersubject variability observed by Wright and Fitzgerald, it may be premature to conclude that cue-specific learning in binaural tasks is restricted to one binaural cue and not another.

The large intersubject variability in thresholds reported for untrained subjects in the current study merits further consideration. Figure 6 shows individual thresholds for 30 subjects corresponding to the upper panels of Fig. 1, in addition to two experienced subjects $\left(\mathrm{S}_{\mathrm{e}} 1\right.$ and $\mathrm{S}_{\mathrm{e}} 3$, plotted to the right of the dashed lines) who ran under those same conditions (ICI $=2 \mathrm{~ms}, 73-\mathrm{dB}$ intensity condition). Top and bottom panels of Fig. 6 show lag- and single-click ITD thresholds, respectively. The abscissa shows subject number and the ordinate represents ITD threshold. Each symbol represents one threshold estimate: ten per subject in the top panel and three per subject in the bottom panel. The asterisks in each panel show the threshold estimates for the last run of each condition. These are the 12th and 13th runs of the experiment for the lag- and single-click conditions, respectively. Note that the last run for each condition and subject sometimes produces the lowest, sometimes the highest, and sometimes middle values of thresholds. Note, in addition, that for the lag-click condition, thresholds span the entire range of interaural delays.

A close inspection, however, reveals across subject variability in overall performance. The arrows shown on the bottom axis of the top panel specify subjects who produced relatively low thresholds across most of their runs. Some of these subjects, however, did produce one or two high- 

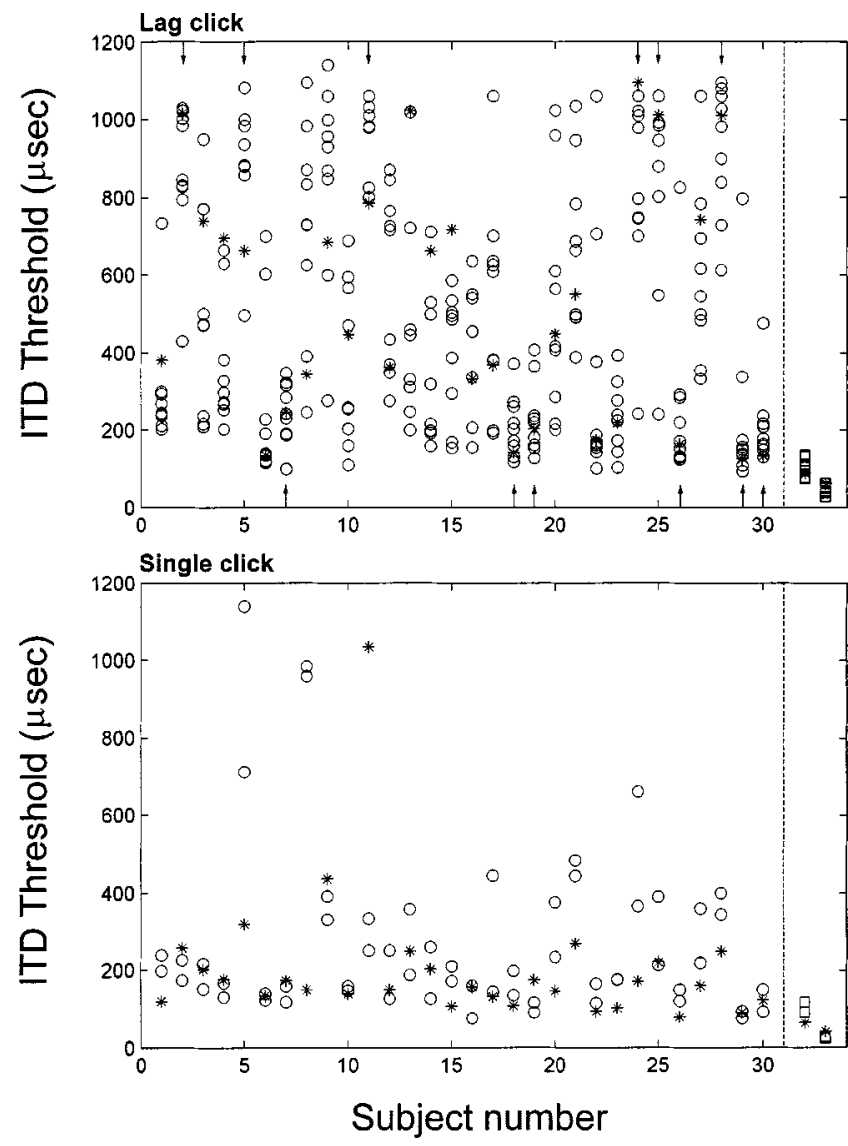

FIG. 6. Single-run thresholds for 30 subjects from the lag- and single-click conditions (top and bottom panels, respectively). Data from untrained subjects correspond to the upper panels of Fig. 1. The ICI was $2 \mathrm{~ms}$ and the stimulus intensity was $73 \mathrm{~dB}$. Data from two experienced subjects are also shown to the right of the dashed lines. Each circle or asterisk represents one threshold estimate. The asterisks are the final run for each subject at each condition (lag or single click). Arrows on the lower axis of the top panel specify subjects with relatively low thresholds, and the arrows on the top axis specify subjects with high thresholds.

threshold runs. For example, subjects 26 and 30 show a single outlier run. There seemed to be no set pattern to the order of occurrence of these outlier runs (i.e., beginning or end of the experimental run). Other subjects whose thresholds were clustered at low ITD values produced two or three high-threshold outliers (e.g., subjects 6, 22, and 29). The single-click thresholds for these subjects are consistently low. Other subjects specified by the arrows on the top axis of the top panel show predominately high lag-click thresholds, and other subjects show highly variable thresholds. For these latter subjects, single-click thresholds are reasonably low, although for a very few cases, thresholds are high or variable. Other studies have also shown large intersubject variability in precedence-effect tasks. For example, thresholds for subjects S7 and S8 from Litovsky et al. (2000) have averaged lag-click thresholds that differ by a factor of $10(\sim 20$ vs. 200 $\mu \mathrm{s}$ at an ICI of $2.35 \mathrm{~ms}$ ).

Thresholds for the two experienced subjects shown in Fig. 6 are consistently low. Although some untrained subjects produce mean lag-click thresholds that approach those of experienced subjects, the average is at least twice as large and show greater within-subject variance. For example, the lowest mean threshold for the 30 untrained subjects in Fig. 6 is produced by subject $18(205 \mu \mathrm{s})$. Subjects 29 and 30 also appear to produce generally low thresholds. Excluding the two high outliers for subject 29 and one outlier for subject 30 , their thresholds remain higher than those obtained from the two experienced subjects whose mean thresholds are 103 and $49 \mu \mathrm{s}$, respectively. The experienced subjects also produced low single-click thresholds as shown in the bottom panel of Fig. 6.

One explanation for outlier thresholds and/or nonmonotonic adaptive tracks reported here may be the pattern of cross-correlation activity generated by dual-impulse stimuli. These patterns are often complex and may result in ambiguous position cues (Zurek and Saberi, 2003; Saberi and Perrott, 1995). Others have also shown that a number of observations related to the precedence effect may be accounted for by examining the complex pattern of cross-correlation activity resulting from dual-pulse stimuli (Saberi and Perrott, 1995; Tollin and Henning, 1998, 1999; Hartung and Trahiotis, 2001; Zurek and Saberi, 2003). The loss of the signal cue may also be related to adaptation (Hafter et al., 1988), or modulation of attention or other cognitive factors (Clifton, 1987; Hartmann and Rakerd, 1989; Blauert and Col, 1991).

Finally, we also observed an effect of stimulus intensity on lag-click thresholds, consistent with Goverts et al. (2000) who showed that the precedence effect is most effective at mid-range stimulus levels. The reduced strength of precedence at high stimulus levels may be related to leveldependent neurophysiological effects such as the widening of the frequency tuning of peripheral auditory filters, saturation of neuronal responses, or changes in phase-frequency response of auditory nerve fibers (Allen, 1983; Ruggero et al., 1992). Goverts et al. have reported that for sensation levels below about 40 and above $50 \mathrm{~dB}$, the strength of the precedence effect is reduced. A similar effect was observed when the stimulus level was held constant and background noise level was increased (Chiang and Freyman, 1998; Goverts et al., 2000). Interestingly, individuals with mild sensory neural hearing loss also show a decline in the strength of the precedence effect (Goverts et al., 2002). At low sensation levels, internal neural noise may affect onset dominance in the same manner as increasing the level of background external noise. The cause of this weakening of the precedence effect when the signal level is close to the noise floor (internal or external) is not clear.

In summary, findings from the current study show that (1) thresholds from untrained subjects displayed large intersubject variability, (2) the best untrained subjects produced mean thresholds near, but not as low as, those obtained from experienced subjects, (3) the one untrained high-threshold subject tested improved with training over a long period of testing, (4) the center-side stimulus design produced lower thresholds than a commutative design, and (5) poor performance may result from uncertainty with regard to the cue for which to listen. Adaptive tracks for untrained subjects were often unstable, consistent with either losing the cue or listening to the wrong cue in an ambiguous stimulus. 


\section{ACKNOWLEDGMENTS}

This work was supported by a Grant from the National Institutes of Health (DC-03648). We thank Dr. David R. Perrott, Dr. Pat Zurek, Agavni G. B. Pertosyan, Kyle Nakamoto, and Prisilia Tirtabudi for helpful comments.

${ }^{1}$ For comparison, a continuous $10-\mathrm{Hz}$ train of clicks had intensities of 51 , 66 , and $81 \mathrm{~dB}$, and at $100-\mathrm{Hz}$ these intensities were 60,75 , and $90 \mathrm{~dB}$. ${ }^{2}$ This idea was suggested by the Associate Editor, Dr. Leslie Bernstein. ${ }^{3}$ Note that the lower threshold was plotted in Fig. 3.

${ }^{4}$ Nearly all naive listeners showed such patterns and the $20 \%$ refers to the approximate proportion of all runs. These were visually categorized by experimenters as the track types described in the text (e.g., U-shaped, single or double peaks, or a sharp trough type).

Allen, J. B. (1983). "Magnitude and phase-frequency response to single tones in the auditory nerve," J. Acoust. Soc. Am. 73, 2071-2092.

Bernstein, L. R., Trahiotis, C., and Hyde, E. L. (1998). "Inter-individual differences in binaural detection of low-frequency or high-frequency tonal signals masked by narrow-band or broadband noise," J. Acoust. Soc. Am. 103, 2069-2078.

Blauert, J. (1971). "Localization and the law of the first wavefront in the median plane," J. Acoust. Soc. Am. 50, 466-470.

Blauert, J. (1989). "Binaural technology: Fundamentals and applications," J. Acoust. Soc. Am. Suppl. 1 86, S66.

Blauert, J. (1997). Spatial Hearing (MIT, Cambridge, MA).

Blauert, J., and Col, J. (1991). "Irregularities in the precedence effect," in Auditory Physiology and Perception: Proceedings of the 9th International Symposium on Hearing, edited by Y. Cazals, L. Demany, and K. Horner (Pergamon, Oxford), pp. 531-538.

Chiang, Y. C., and Freyman, R. L. (1998). "The influence of broadband noise on the precedence effect," J. Acoust. Soc. Am. 104, 3039-3047.

Clifton, R. K. (1987). "Breakdown of echo suppression in the precedence effect," J. Acoust. Soc. Am. 82, 1834-1835.

Clifton, R. K., and Freyman, R. L. (1989). "Effect of click rate and delay on breakdown of the precedence effect," Percept. Psychophys. 46, 139-145.

Cranford, J., and Oberholtzer, M. (1976). "Role of neocortex in binaural hearing in the cat: II. The 'precedence effect' in sound localization," Brain Res. 111, 225-239.

Djelani, T., and Blauert, J. (2001). "Investigations into the build-up and breakdown of the precedence effect," Acustica 87, 253-261.

Franssen, N. V. (1960). "Some considerations on the mechanism of directional hearing," Ph.D. thesis, Technische Hogeschool, Delft, The Netherlands.

Freyman, R. L., Zurek, P. M., Balakrishnan, U., and Chiang, Y.-C. (1997). "Onset dominance in lateralization," J. Acoust. Soc. Am. 101, 16491659.

Gardner, M. B. (1968). "Historical background of the Haas and/or precedence effect," J. Acoust. Soc. Am. 43, 1243-1248.

Gaskell, H. (1983). "The precedence effect," Hear. Res. 12, 277-303.

Goverts, S. T., Houtgast, T., and van Beek, H. H. M. (2000). "The precedence effect for lateralization at low sensation levels," Hear. Res. 148, $88-94$.

Goverts, S. T., Houtgast, T., and van Beek, H. H. M. (2002). "The precedence effect for lateralization for the mild sensory neural hearing impaired," Hear. Res. 163, 82-92.

Haas, H. (1949). "The influence of a single echo on the audibility of speech,” J. Audiol. Eng. Soc. 20, 145-159, English translation (1972).

Hafter, E. R., and Carrier, S. C. (1970). "Masking level differences obtained with pulsed tonal maskers," J. Acoust. Soc. Am. 47, 1041-1047.

Hafter, E. R., and Dye, R. H. (1983). " Detection of interaural differences of time in trains of high-frequency clicks as a function of interclick interval and number," J. Acoust. Soc. Am. 73, 1708-1713.

Hafter, E. R., Buell, T. N., and Richards, V. M. (1988). "Onset-coding in lateralization: Its form, site, and function," in Auditory Function, edited by G. M. Edelman, W. E. Gail, and W. M. Cowan (Wiley, New York).

Hafter, E. R., Dye, R. H., and Wenzel, E. (1983). "Detection of interaural differences of intensity in trains of high-frequency clicks as a function of interclick interval and number," J. Acoust. Soc. Am. 73, 644-651.

Hartmann, W. M., and Rakerd, B. (1989). "Localization of sound in rooms. 4. The Franssen effect,” J. Acoust. Soc. Am. 86, 1366-1373.
Hartung, K., and Trahiotis, C. (2001). "Peripheral auditory processing and investigations of the 'precedence effect' which utilize successive transient stimuli," J. Acoust. Soc. Am. 110, 1505-1513.

Hays, W. L. (1981). Statistics, 2nd ed. (CBS College, New York).

Hochster, M. E., and Kelly, J. B. (1981). "The precedence effect and sound localization by children with temporal lobe epilepsy," Neuropsychologia 19, 49-55.

Leakey, D. M. (1957). "Some measures on the effects of interchannel intensity and time differences in two channel sound systems," J. Acoust. Soc. Am. 31, 977-986.

Levitt, H. (1971). "Transformed up-down methods in psychoacoustics," J. Acoust. Soc. Am. 49, 467-477.

Lindemann, W. (1986a). "Extension of a binaural cross-correlation model by contralateral inhibition: I. Simulation of lateralization for stationary signals," J. Acoust. Soc. Am. 80, 1608-1622.

Lindemann, W. (1986b). "Extension of a binaural cross-correlation model by contralateral inhibition: II. The law of the first wavefront," J. Acoust. Soc. Am. 80, 1608-1622.

Litovsky, R. Y., Colburn, H. S., Yost, W. A., and Guzman, S. J. (1999). "The precedence effect," J. Acoust. Soc. Am. 106, 1633-1654.

Litovsky, R. Y., Hawley, M. L., Fligor, B. J., and Zurek, P. M. (2000). "Failure to unlearn the precedence effect," J. Acoust. Soc. Am. 108, $2345-2352$.

Mickey, B. J., and Middlebrooks, J. C. (2001). "Responses of auditory cortical neurons to pairs of sounds: Correlates of fusion and localization," J. Neurophysiol. 86, 1333-1350.

Muncey, R. W., Nickson, A. F. B., and Dubout, P. (1953). "The acceptability of speech and music with a single artificial echo," Acustica 3, 168-173.

Perrott, D. R., Marlborough, K., Merrill, P., and Strybel, T. Z. (1989). "Minimum audible angel thresholds obtained under conditions in which the precedence effect is assumed to operate," J. Acoust. Soc. Am. 85, 282-288.

Pitman, J. (1993). Probability (Springer Verlag, New York).

Rakerd, B., Hartmann, W. M., and Hsu, J. (2000). "Echo suppression in the horizontal and median sagittal planes," J. Acoust. Soc. Am. 107, 10611064

Ruggero, M. A., Rich, N. C., and Recio, A. (1992). "Basilar membrane responses to clicks," in Auditory Physiology and Perception, edited by Y. Cazals, L. Demany, and K. Horner (Pergamon, Oxford), pp. 85-92.

Saberi, K. (1995). "Some considerations on the use of adaptive methods for estimating interaural-delay thresholds," J. Acoust. Soc. Am. 98, 18031806.

Saberi, K. (1996). "Observer weighting of interaural delays in filtered impulses," Percept. Psychophys. 58, 1037-1046.

Saberi, K., and Perrott, D. R. (1990). "Lateralization thresholds obtained under conditions in which the precedence effect is assumed to operate," $\mathrm{J}$. Acoust. Soc. Am. 87, 1732-1737.

Saberi, K., and Perrott, D. R. (1995). "Lateralization of click-trains with opposing onset and ongoing interaural delays," Acustica 81, 272-275.

Shinn-Cunningham, B. G., Zurek, P. M., and Durlach, N. I. (1993). "Adjustment and discrimination measurements of the precedence effect," $\mathrm{J}$. Acoust. Soc. Am. 93, 2923-2032.

Shinn-Cunningham, B. G., Zurek, P. M., Durlach, N. I., and Clifton, R. K. (1995). "Cross-frequency interactions in the precedence effect," J. Acoust. Soc. Am. 98, 164-171.

Tollin, D. J., and Henning, G. B. (1998). "Some aspects of the lateralization of echoed sound in man. I. The classical interaural-delay based precedence effect," J. Acoust. Soc. Am. 104, 3030-3038.

Tollin, D. J., and Henning, G. B. (1999). "Some aspects of the lateralization of echoed sound in man. II. The role of stimulus spectrum," J. Acoust. Soc. Am. 105, 838-849.

Wallach, H., Newman, E. B., and Rosenzweig, M. R. (1949). "The precedence effect in sound localization," Am. J. Psychol. 52, 315-336.

Wetherill, G. B., and Levitt, H. (1965). "Sequential estimation of points on a psychometric function," Br. J. Math. Stat. Psychol. 18, 1-10.

Wright, B. A., and Fitzgerald, M. B. (2001). "Different patterns of human discrimination learning for two interaural cues to sound-source location," Proc. Natl. Acad. Sci. U.S.A. 98, 12307-12312.

Yin, T. C. T., and Litovsky, R. Y. (1995). "Physiological studies of the precedence effect in the inferior colliculus of the cat," in Advances in 
Hearing Research, edited by G. A. Manley, G. M. Klump, C. Koppl, H. Fastl, and H. Oeckinghaus (World Scientific, Singapore).

Yost, W. A., and Soderquist, D. R. (1984). "The precedence effectrevisited," J. Acoust. Soc. Am. 76, 1377-1383.

Zurek, P. M. (1980). "The precedence effect and its possible role in the avoidance of interaural ambiguities," J. Acoust. Soc. Am. 67, 952-964. Zurek, P. M. (1987). "The precedence effect," in Directional Hearing, edited by W. A. Yost and G. Gourevitch (Springer-Verlag, New York).

Zurek, P. M., and Saberi, K. (2003). "Lateralization of two-transient stimuli," Percept. Psychophys. 65, 95-106. 